\title{
3D simulations of the experimental signal measured in near-field optical microscopy
}

\author{
O. J. F. MARTIN \\ Electromagnetic Fields and Microwave Electronics Laboratory, Swiss Federal Institute of \\ Technology, ETH-Zentrum, 8092 Zurich, Switzerland
}

Key words. Artefacts, contrast mechanisms, image formation, local probe microscopy, near-field optics, polarization, simulations, theory, topography.

\section{Summary}

We present three-dimensional simulations of the image formation process in near-field optical microscopy. Our calculations take into account the different components of a realistic experiment: an extended metal coated tip, a subwavelength sample and its substrate. We investigate all possible detection (transmitted, reflected and collected field) and scanning (constant height, constant gap) modes. Our results emphasize the strong influence of the tip motion on the experimental signal. They also show that it is possible, by controlling the polarization of both the illumination and the detected field, to strongly reduce these artefacts.

\section{Introduction and model}

Over the last few years, scanning near-field optical microscopy (SNOM) has become an increasingly useful tool in many domains of applied science, ranging from solid state physics to analytical chemistry and biology (for a recent overview see the special issue of Ultramicroscopy, 71, 1-4 (1998)).

In spite of this strong experimental interest, the theoretical understanding of SNOM images remains somewhat unsatisfactory (see for example the revue articles by Girard \& Dereux (1996) and by Greffet \& Carminati (1997) that describe the state-of-the-art in that matter).

This is probably due to the complexity and the diversity of effects that are at play in SNOM. Indeed, while at first sight one thinks that the entire image formation process is similar to scanning a dipole above a small object, one rapidly realizes that this process is much more complex.

In particular, the interaction of the tip with the sample and its influence on the signal detected in the far-field is

Correspondence to: O. J. F. Martin, E-mail: martin@ifh.ee.ethz.ch rather intricate. A typical SNOM configuration is depicted in Fig. 1. The tip is raster-scanned above a sample deposited on a transparent substrate.

The tip motion can be effectuated either in constant height mode or in constant gap mode. In constant height, the tip is kept at a fixed altitude above the substrate, while in constant gap mode it follows the topography at a fixed distance.

Different detection modes are possible: transmitted light, reflected light and collected light. In this last mode, the tip is used both to illuminate and detect the signal.

For the transmitted light, one can distinguished between allowed and forbidden light, the latter been transmitted in the substrate at angles larger than the total internal reflection angle for that system.

For brevity we don't consider here separately constant intensity mode, since it was proven similar to constant height mode by Greffet \& Carminati (1997). From a theoretical point of view, it is desirable to compute these different signals as a function of the tip position while it raster scans the sample.

In this paper we use the Green's tensor technique to investigate this problem. This technique has been described extensively elsewhere (Martin \& Piller, 1998) and we will only describe it briefly here. The key feature is that this technique can easily accommodate a complex background, like the semi-infinite substrate in Fig. 1, by using the appropriate Green's tensor (Girard et al., 1995). Like this, only the geometrical elements that do not belong to the background must be discretized to build up the system of equations to be solved numerically for the scattered field. For the practical case of Fig. 1, only the tip and the defect must then be discretized.

As illumination, we use the corresponding evanescent field distribution of a metal coated fibre below cutoff (Novotny \& Hafner, 1994). For all the results presented here, the illumination wavelength in vacuum is $633 \mathrm{~nm}$ and the polarization of the incident field is in $x$-direction. 


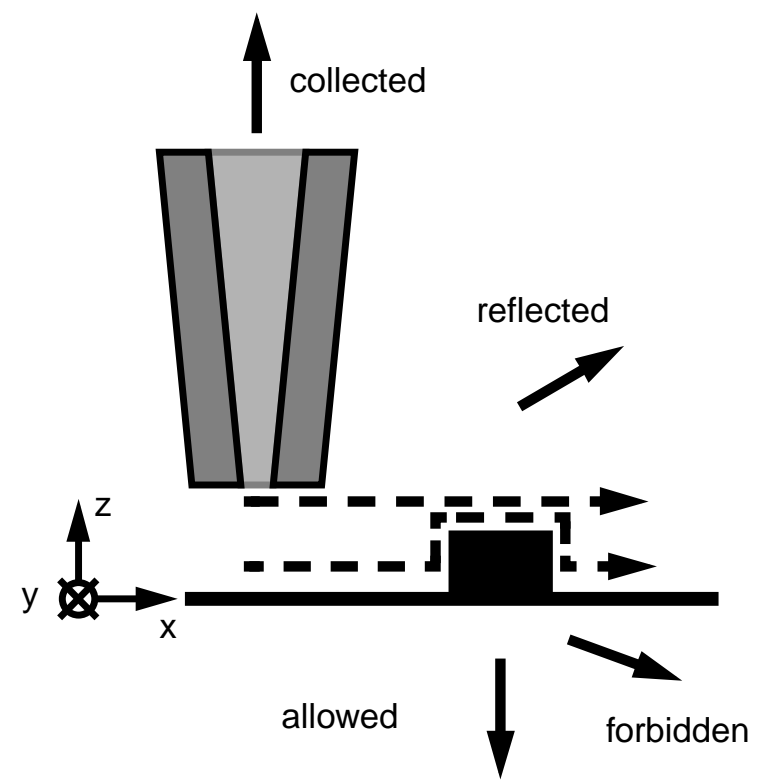

Fig. 1. Geometry of the system. The sample under study is placed on a transparent surface and raster-scanned with a metal-coated tip, either at constant height or at constant gap. Four different detection modes are investigated: detection in the far-field (allowed, forbidden and reflected light) and in the near-field (collected light).

For the tip, we use a geometry similar to that obtained for etched tips (Lambelet et al., 1998; Sayah et al., 1998; Stöckle et al., 1998). The opening angle of the glass core is $20^{\circ}$ and the aluminium coating $70 \mathrm{~nm}$ thick. The tip apex forms a flat plateau with a $50 \mathrm{~nm}$ diameter glass opening surrounded by the coating disc; the total diameter of the tip apex being $190 \mathrm{~nm}$. The substrate and the sample are made of glass $(\varepsilon=2)$. The sample dimensions are $40 \times 40 \times 40 \mathrm{~nm}^{3}$.

\section{Results}

Although our calculations are fully 3D, we only present line scans along two orthogonal directions. It should be emphasized that each point of a line scan corresponds to a complete self-consistent calculation for a particular tip position. Our aim is to investigate the topology of the signal measured in a realistic experiment for different scanning or detection modes. We therefore normalize all our results in a similar way, so that they can be easily compared.

Anyhow, a quantitative prediction for the absolute intensity of the experimental signal is difficult, since it strongly depends on the sensitivity of the detection equipment and on the detection mode. In particular, it is obvious that in collection mode, the signal is much smaller that in transmission mode, since the light must pass twice through the aperture.

\section{Constant height mode}

The main difficulty in the interpretation of near-field images is the strong coupling between the topography and the optical signal (Hecht et al., 1997). To investigate optical contrast, we therefore first keep the scanning height constant above the substrate so that the tip apex passes $5 \mathrm{~nm}$ above the sample. In Fig. 2, we report the transmitted intensity as a function of the tip position for two orthogonal scanning directions. The difference of behaviour observed in this figure is easily understood if we recall that the incident field is polarised in $x$-direction. Therefore, when scanning in the same $x$-direction, the electric field is normal to the different material interfaces in the system. To satisfy the boundary conditions imposed by Maxwell equations, which require the normal displacement field to be continuous, a strong depolarization field must be created at these interfaces. This depolarization field produces an important near-field signal (Martin et al., 1996). Although a minimum is visible for the $x$-scan in Fig. 2, the resolution remains in the order of the overall tip diameter.

Scanning perpendicularly to the incident polarization ( $y$ scan, Fig. 2) leads to a relatively different signal. This is because no depolarization effects occur during this scan since the field remains parallel to the different material interfaces. We observe that the resolution in this direction is in the order of the aperture diameter. Let us emphasize that in a SNOM experiment, one does not take only line scans,

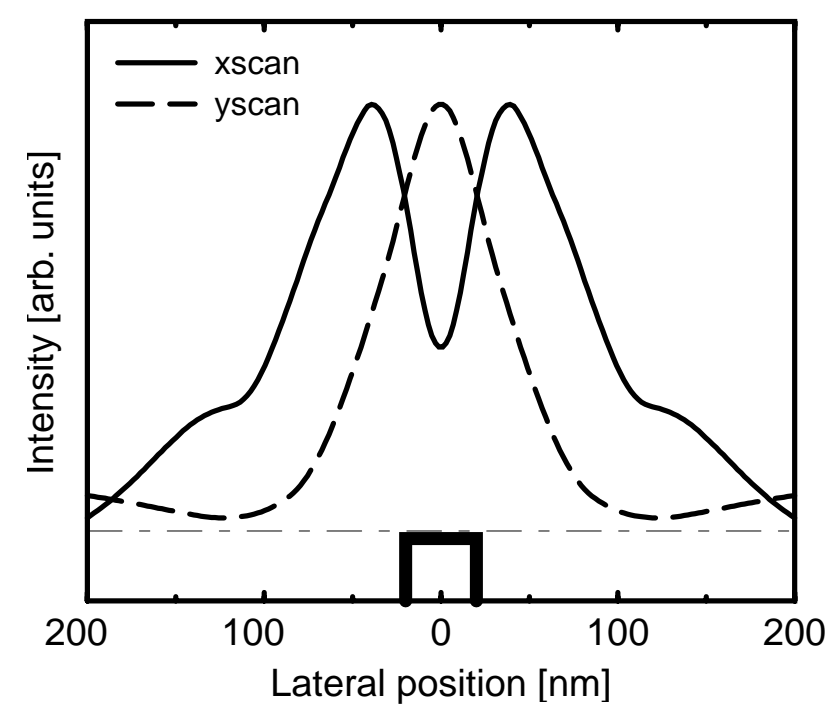

Fig. 2. Transmitted light intensity for two different scan directions. Constant height scans (the dot-dashed line represents the position of the apex centre during the scan). 
but records complete images. Our results imply that in such an image, the sides of an object will be highlighted differently, depending on their relative orientation to the incident polarization.

The signals measured with other 'external' detection modes for the same configuration are reported in Fig. 3. Note the contrast reversal between allowed and forbidden light, which is also observed experimentally (see, e.g. Hecht et al., 1995).

A very interesting detection mode is collection mode, where the intensity is measured through the same aperture. Although this mode is difficult experimentally because it produces extremely small signals, it is the only one that provides both localized illumination and localized detection. Signals measured with this detection mode are given in Fig. 4 for scans in the $x$-direction. Let us first concentrate on the total field (Fig. 4(a)). These results show more oscillations than in the previous cases. We see both the signature of the overall tip (diameter $\approx 200 \mathrm{~nm}$ and of the aperture (diameter $50 \mathrm{~nm}$ ) in the signal. The physical origin of the ripples observed in Fig. 4(a) becomes obvious when one discriminates the polarization of the collected field (Fig. 4(b)). We then observe that each material interface produces a sharp peak in the collected signal. This behaviour is similar for both polarizations.

\section{Constant gap mode}

Let us now turn to the other scanning mode: constant gap, where the tip follows the topography of the system. We choose a $2 \mathrm{~nm}$ separation between the tip apex and the system.

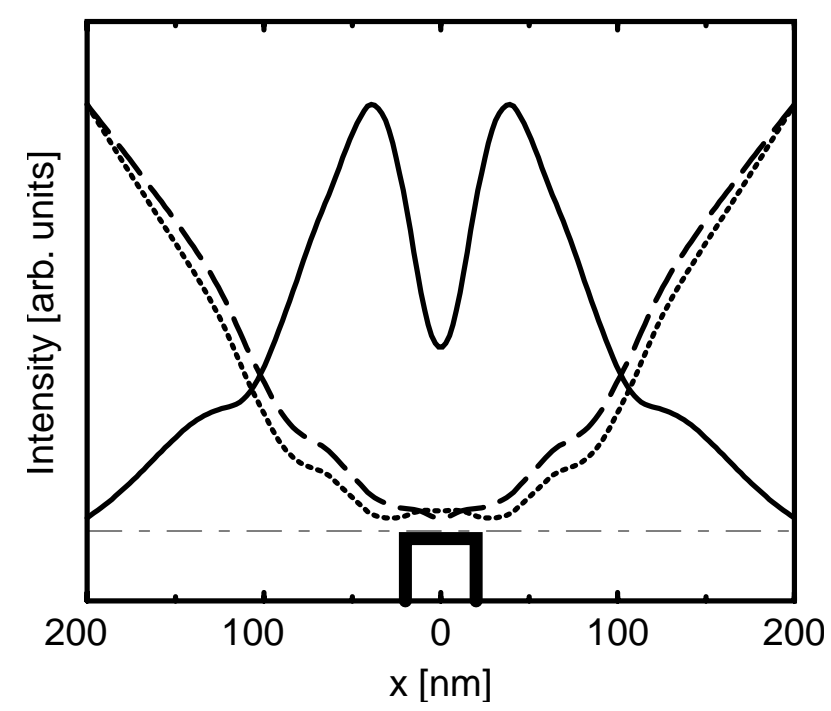

Fig. 3. Signal intensity for three different detection modes: allowed light (continuous line), forbidden light (dashed line) and reflected light (dotted line). Constant height scans in $x$-direction.
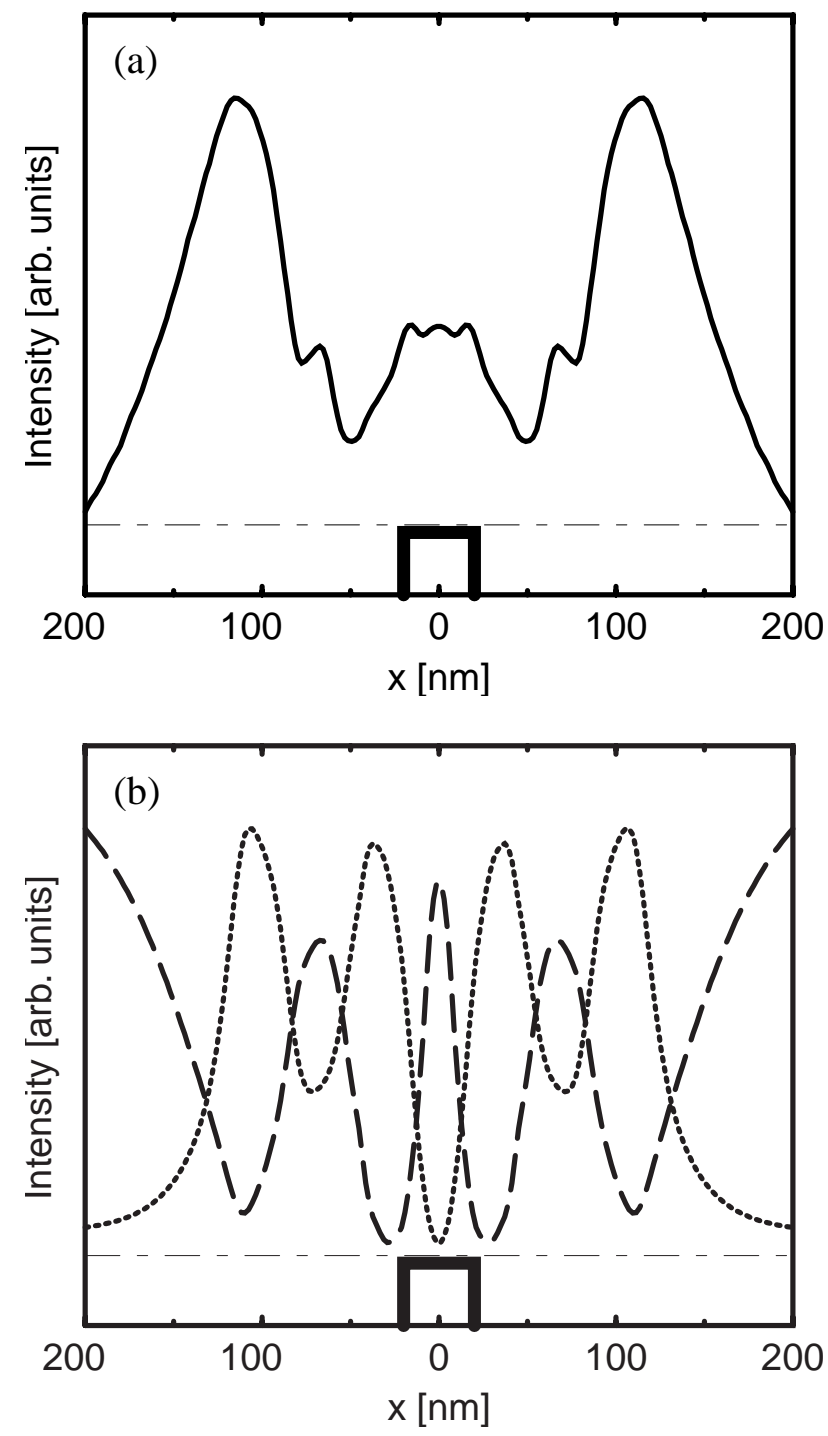

Fig. 4. Signal intensity in collection mode. (a) Total field and (b) polarization discriminated field: $x$-polarization (dashed line) and $y$-polarization (dotted line). Constant height scans in $x$-direction.

The trajectory of the apex centre is given in each figure. This trajectory is determined by the width and the shape of the tip. Figure 5 reports the intensity collected for two scans in $x$ - and $y$-directions. Note the strong correlation between the motion of the tip and the near-field signal (compare with Fig. 2). This is a good illustration of the artefacts that can jeopardize optical images, as was pointed out recently by Hecht etal. (1997). As a matter of fact, in an image produced this way, strong bright spots would appear on the side of the object, where the tip starts its ascent. Although related to the topography of the object, theses peaks do not correspond to a strong localized field at the vicinity of the sample, but 


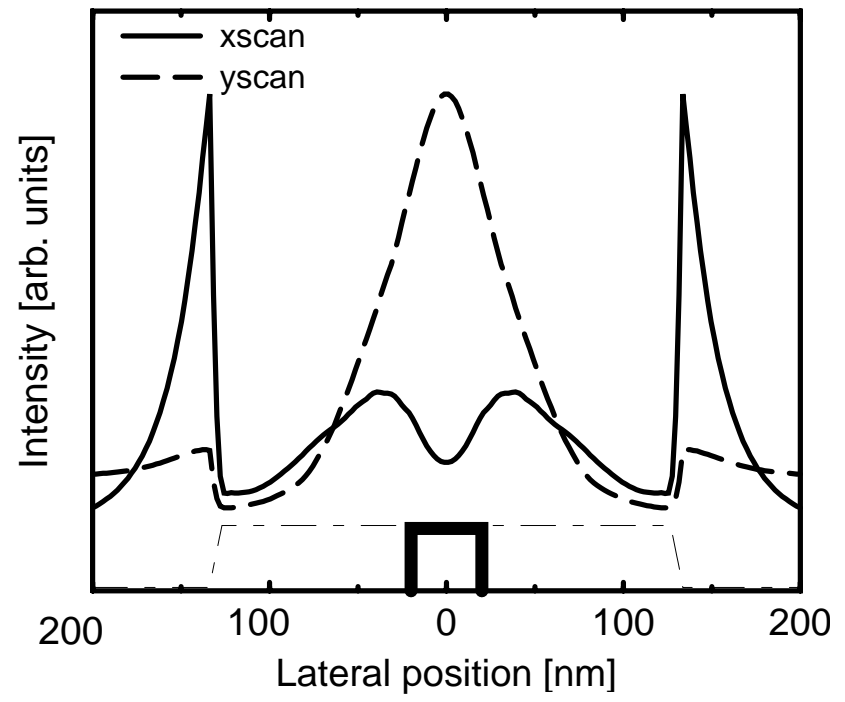

Fig. 5. Transmitted light intensity for two different scan directions. Constant gap scans (the dot-dashed line represents the position of the apex centre during the scan). Compare with Fig. 2, where the scans are performed at constant height.

merely to the variation of the distance between the tip and the substrate.

Also in Fig. 5 the difference between the scan directions is striking. Indeed, although the tip follows a completely similar path to trace the defect outline, the signal induced by the tip motion is much more important for the $x$-scan than for the $y$-scan. This is again related to depolarization effects that occur on the object sides during the upward tip motion and that are more pronounced when the incident field is normal to the object side ( $x$-scan).

These results show that topographic artefacts can be even more insidious than one might think since they are not only determined by the tip motion, but also depend on the polarization of the field. This also emphasizes the importance of fully 3D calculations for analysing image formation in near-field optical microscopy. A similar behaviour is observed for the other external detection modes (not shown), where strong variations of the signal correlated to the vertical tip motion are also measured.

In collection mode, the influence of the topography on the measured near-field signal is particularly strong, as illustrated in Fig. 6(a). We see that the total field intensity simply reproduces the tip motion and any optical information is lost. We believe that this is caused by the dominating interaction between the tip and the surface in that mode. On the other hand, if one controls the polarization of the collected field, one records a signal that does not suffer from artefacts related to the tip motion (Fig. 6(b)). A similar behaviour is observed if one collects the cross-polarised signal ( $y$-polarization).
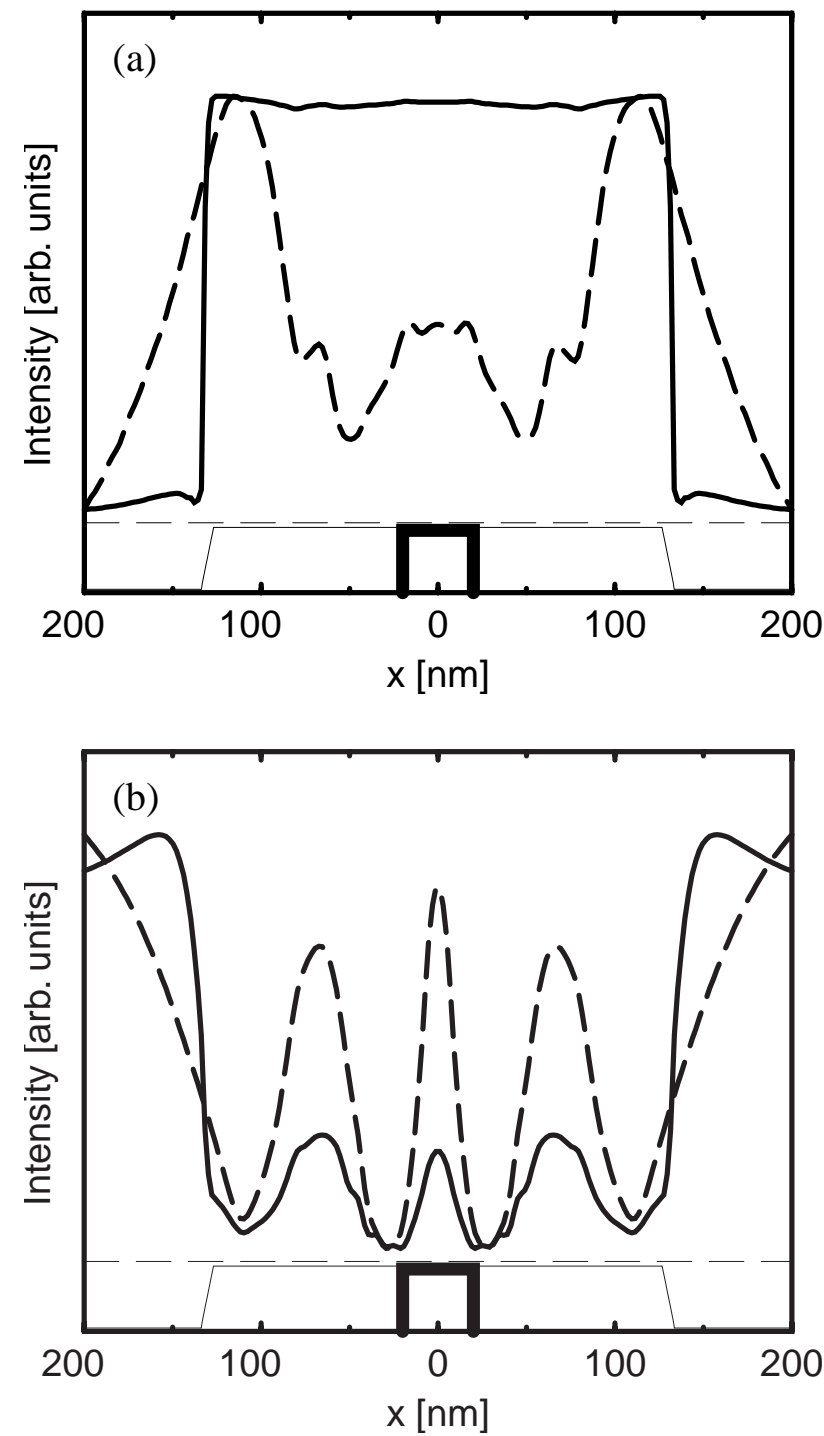

Fig. 6. Comparison of constant gap (continuous line) and constant height (dashed line) scan modes. Collected light intensity (a) total field and (b) $x$-polarised field. The corresponding trajectory of the apex centre is reported at the bottom of the figures.

\section{Conclusion}

The 3D calculations presented in this paper illustrate the complexity of image formation in near-field microscopy. They emphasize the strong correlation between the tip motion and the total field intensity detected in the far-field (transmission, reflection modes) and in the near-field (collection mode). On the other hand, it appears that an accurate control of the polarization both for the illumination and the detected field allows one to reduce these artefacts. To make the physical interpretation of images possible, it is mandatory in on-going experiments to keep the field polarization under control. Furthermore, realizing two successive scans with cross-polarization for the illumination field should enhance the optical information. 


\section{Acknowledgements}

This work was supported by the Swiss National Science Foundation. It is a pleasure to acknowledge stimulating discussions with B. Hecht and D. W. Pohl.

\section{References}

Girard, C. \& Dereux, A. (1996) Near-field optics theories. Reports Prog. Phys. 59, 657-699.

Girard, C., Dereux, A., Martin, O.J.F. \& Devel, M. (1995) Generation of optical standing waves around mesoscopic surface structures: scattering and light confinement. Phys. Rev. B, 52, 2889-2898.

Greffet, J.-J. \& Carminati, R. (1997) Image formation in near-field optics. Prog. Surf. Sci. 56, 133-237.

Hecht, B., Bielefeld, H., Inouye, Y., Pohl, D.W. \& Novotny, L. (1997) Facts and artifacts in near-field optical microscopy. J. Appl. Phys. 81, 2499-2503.

Hecht, B., Pohl, D.W., Heinzelmann, H. \& Novotny, L. (1995)
'Tunnel' near-field optical microscopy. TNOM-2. Ultramicroscopy, 61, 99-104.

Lambelet, P., Sayah, A., Pfeffer, M., Philipona, C. \& Marquis-Weible, F. (1998) Chemically etched fiber tips for near-field optical microscopy: a process for smoother tips. Appl. Opt. 37, 7289-7292.

Martin, O.J.F., Girard, C. \& Dereux, A. (1996) Dielectric versus topographic contrast in near-field microscopy. J. Opt. Soc. Am. A, 13, 1801-1808.

Martin, O.J.F. \& Piller, N.B. (1998) Electromagnetic scattering in polarizable backgrounds. Phys. Rev. E, 58, 3909-3915.

Novotny, L. \& Hafner, C. (1994) Light propagation in a cylindrical waveguide with a complex, metallic, dielectric function. Phys. Rev. E, 50, 4094-4106.

Sayah, A., Philipona, C., Lambelet, P., Pfeffer, M. \& MarquisWeible, F. (1998) Fiber tips for scanning near-field optical microscopy fabricated by normal and reverse etching. Ultramicroscopy, 71, 59-63.

Stöckle, R., Fokas, C., Deckert, V., Zenobi, R., Sick, B., Hecht, B. \& Wild, U.P. (1998) Tube-etching: towards ideal near-field optical probes. Appl. Phys. Lett., submitted. 